\title{
Dealing with fuzzy ontology integration problem by using constraint satisfaction problem
}

\author{
Xuan Hung Quach* and Thi Lan Giao Hoang \\ College of Sciences, Hue University, Vietnam \\ Received: 08-December-2016; Revised: 27-February-2017; Accepted: 05-March-2017 \\ (C)2017 ACCENTS
}

\begin{abstract}
The problem of fuzzy ontology integration can be divided into two phases: the matching phase between ontologies and conflicting resolution on fuzzy values. This paper focuses on the problems of the matching phase, which currently has exhaustive and heuristic approaches. While the exhaustive method has many matching errors, most of the matched pairs between the two ontologies are detected. The heuristic approach uses the ontology nature to trim the non-homologous pairs which can decrease significantly the number of mismatching pairs, but often skips lots of homologous element pairs. To overcome the disadvantages of the two approaches, we proposed to use the constraint satisfaction problem (CSP) for modeling the ontology matching problem. In particular, we introduce constraints and suggest optimal function to minimize the matching errors. In the experiment, the mismatching pairs between ontologies are significantly reduced by applying CSP for refinement.
\end{abstract}

\section{Keywords}

Ontology, Ontology integration, Ontology matching, Constraint satisfaction problem, Constraint optimization problem.

\section{Introduction}

In distributed knowledge systems, there always exists information heterogeneity which is necessarily addressed when the system is required to exchange, share or reuse either information or data. Ontology has been considered as the cornerstone of the knowledge-based information system. Thus, in order to use and deploy this system, multi-ontology integration is a crucial task. However, it is indeed a complicated issue because of the information heterogeneity as well as diversified structures. Knowledge integration is a process in which the inconsistency of knowledge from different sources is consolidated to provide a knowledge unity. Recent studies on ontology integration are conducted with different levels: ontology matching, ontology alignment, ontology merges, ontology mapping. The concept of ontology integration is defined as [1]: given $\mathrm{n}$ ontologies $O_{1}, \ldots O_{n}$, ontology integration is to find out a new ontology $\mathrm{O}^{*}$ best from $O_{1}, \ldots O_{n}$ ones.

*Author for correspondence
According to the ontology integration researches, it is necessary to model ontology first by utilizing description logic and then study the equivalent mathematical operations between ontology elements. Besides, strategy or integration approach is also a big deal, which needs much concern.

Currently, the crisp ontology concept relying on conventional description logic is quite limited because it cannot afford to represent vague and uncertain information. For example: conventional weather ontology could not represent whether the quantitative property value - such as rainfall or sunshine - in a day is more or less. Other examples are: "a funny holiday in a sea" and "at a very cold place" that the adverb and adjective usage on semantic web could not be well-defined on crisp ontology. It might be tough to understand how much the "funny" or "very cold" value is. As a result, this leads to the appearance of more and more research on the fuzzy ontology field that researchers focus on tackling with the information vagueness. The current crisp ontology integration techniques are not suitable and even too few practical methods for fuzzy ontology integration. Fuzzy ontology matching [2] can be classified into two types: The first type is an extension of crisp ontology matching approach and 
the second one is a way of resolving fuzziness among concepts during the matching execution. Rung-Ching Chen [3] and his colleagues proposed a mixed ontology approach combining WordNet and fuzzy formal concept analysis (FFCA) [3] to produce a fuzzy ontology called mixed FFCA. Recently, the research group of Professor Nguyen Ngoc Thanh has proposed some solutions for handling fuzzy ontology contradiction as reported in these papers [1, 4-7]. We also studied the fuzzy ontology integration relying on consensus theory [8] and resolved the conflict between the different levels of the fuzzy ontologies comprising concept degree, relationship degree and reality degree. However, most of these methods are ceased at fuzziness handling after matching. In this work, we propose a general model for integrating multiple ontologies by employing CSP, utilizing CSP and objective function for the matching process optimization.

\section{Fuzzy ontology definition}

We propose a fuzzy ontology model in which the components are fuzzed by using a membership function. A fuzzy ontology model is proposed by integrating fuzzy logic and fuzzy description logic. This fuzzy ontology definition is an extension of the earlier proposed ontology [8]:

\subsection{Definition 1: Fuzzy ontology}

Let $(\mathrm{A}, \mathrm{V})$ be a real world, where $\mathrm{A}$ is a finite set of attributes, V is value domain of A. Fuzzy Ontology is determined by a set of four parameters $(C, R, I, Z)$, in which:

-C is a finite set of fuzzy concept. $\mathrm{C}=\left\{\left(c_{i}, f^{C}\left(c_{i}\right)\right)\right.$ $\mid \mathrm{i}=1 \ldots \mathrm{n}\}$, where $f^{C}\left(c_{i}\right)$ is the value of membership function $f^{C}$ of the concept $c_{i} \in C, f^{C}\left(c_{i}\right) \in[0,1]$.

-R is a set of fuzzy relations between concepts $\mathrm{R}=$ $\left\{R_{1}, R_{2}, \ldots, R_{m}\right\}, R_{i} \subseteq \mathrm{C} \times \mathrm{C} \times[0,1], \mathrm{i}=1 \ldots \mathrm{m} . \mathrm{A}$ relation is a set having a pair of concepts and fuzzy value representing their correlations. The relation $R_{i}$ between two concepts in an ontology, represented by a unique fuzzy value, means that

-if (c, c', v) $\in R_{i}$ and (c, c', v') $\in R_{i}$ then $\mathrm{v}=\mathrm{v}^{\prime}$. -I is a set of fuzzy entities, $\mathrm{I}=\left\{I_{1}, I_{2}, \ldots, I_{k}\right\}, I_{i}=$ $\left\{\left(i, f^{I_{i}}(i)\right) \mid i \in c_{i}\right\}$, in which $f^{I_{i}}(i)$ is the value of membership function $f^{I_{i}}$ of case $i \in c_{i}, f^{I_{i}}(i) \in[0,1]$

$-\mathrm{Z}$ is a set of axioms, the integrity constraints or relationships between concepts and entities in ontology. They cannot be represented by the relationships in $\mathrm{R}$ or the conditions (necessary and sufficient) to determine the concept $\mathrm{C}$.

82

\subsection{Definition 2: Specific fuzzy concept}

The specific fuzzy concept $c f \subset C$ is defined as a set of five parameters: $\left(c f, V_{c f}, V_{c f}^{\prime}, L_{c f}, f_{c f}\right)$ where cf is a unique identifier. $V_{c f} \subset V$ is a specific set, called a value domain. $V_{c f}^{\prime} \subset[0,1]$ demonstrates the fuzzy values of the specific set $V_{c f}$. $f_{c f}$ is a fuzzy membership function, determined by: $f_{c f}: V_{c f} \rightarrow$ $V_{c f}^{\prime}$, with $\forall v \in V_{c f}, f_{c f}(v) \in V_{c f}^{\prime} . \quad L_{c f} \subset V$ is the language value to determin attribute value level in $V_{c f}$.

An example of a specific fuzzy concept is: Nil, Trace, Light, Moderate, and Heavy to describe the fuzzy concept rainfall.

\subsection{Definition 3: Instance}

An instance of a concept $\mathrm{c}$ is described by the attribute of set $A^{c}$, with the values in set $V^{c}$. The concept $\mathrm{c}$ is a pair (id, v), in which id is the identifier of the instance and $v$ is the value the mapping instance $v: A^{c} \rightarrow V^{c}, v(a) \in V^{c}, \forall a \in A^{c}$.

\subsection{Definition 4: The specific fuzzy attribute}

The specific fuzzy attribute $\mathrm{d}$ is defined as a pair: $d=$ $\left(c_{d}, c_{r}\right)$, or set of five parameters: $d=\left(c_{d}, V_{d}, V_{d}^{\prime}, L_{d}, f_{d}\right)$. Where, $\mathrm{d}$ the unique identifier of the attribute. $c_{d} \in C$ is a fuzzy concept called attribute domain $\mathrm{d}, c_{r}$ is the specific fuzzy concept. $V_{d} \subset V$ is a specific set called value range domain d. $V_{d}^{\prime} \subset[0,1]$ present the fuzzy values of the specific set $V_{d} . f_{d}$ is the fuzzy membership function, determined by:

$f_{d}: V_{d} \rightarrow V_{d}^{\prime}$, vó $i \forall v \in V_{d}, f_{d}(v) \in V_{d}^{\prime} . L_{d} \subset V$ is the language value to determine attribute value level in $V_{d}$.

Example: the concept Rain \{hasRainfall, hasDepth has two specific fuzzy attributes hasRainfall and hasDepth, where hasRainfall has the value range $(0$, 100], hasDepth has the value range Nil, Trace, Light, Moderate and Heavy.

\subsection{Definition 5: Fuzzy concept}

A concept of fuzzy ontology is defined as a set of four parameters: $\left(c_{i}, A^{c_{i}}, V^{c_{i}}, f^{c_{i}}\right)$, where $c_{i}$ is a set of objects (objects) or representation (instances), $A^{c_{i}} \subseteq \mathrm{A}$ is a set of concept description, $V^{c_{i}} \subseteq \mathrm{V}$ is the value domain of attribute $V^{c_{i}}=\mathrm{U}_{a \in A} V_{a}\left(\mathrm{~V}_{a}\right.$ is the value domain of attribute a) and $f^{c_{i}}$ is a fuzzy membership function: $\quad f^{c_{i}}: \quad A^{c_{i}} \rightarrow[0,1]$ represents the important/determined degree of an attribute to a 
concept $c_{i}$. Set $\left(A^{c_{i}}, V^{c_{i}}, f^{c_{i}}\right)$ is the fuzzy structure of $c_{i}$.

\section{Similarity measurement between ontology components}

One of the most fundamental issues in the process of integration is to find the similarity between ontology components. The similarity between components is conducted by various matching methods and should be employed together effectively. The key point of this matching technique is to choose suitable methods and combine them in the most appropriate way. According to the approach in [5], we propose the similarity degree determination into four groups:

- Similarity degree based on entities: the similarity between the concepts is defined by the general entities.

- Similarity degree based on vocabulary: the similarity between the concepts is defined by the linguistic meaning of the related names.

- Similarity degree based on schema: the similarity between two concepts relies on the similarity between the correlative attributes.

- Similarity degree based on classification: the similarity between two concepts is determined by their structural similarity relationship.

In the article [9], the authors specify two tasks of computing fuzzy similarity on ontology components. The first task is to measure the route distance between two concepts of topology. The second one relies on shared information from the most common and particular node of the two concepts. This method is considered as an extension of the same proportion measurement model composed by Tversky.

Determining the level, semantic similarity, semantic gap, or semantic relationship between concepts from the system as well as different fields, increasingly becomes an important task. This paper presents an overview of the measurements between the concepts of ontology and provide some examples of metrics presented in the studies.

Recently, researchers have used fuzzy ontology to search information [10, 11] and merged multiple ontologies to build a common knowledge structure [11]. There are some specific examples of determining the similarity level and distance measurement between ontologies used in the fuzzy ontology. One of the natural methods [12] to determine the semantic similarity is to use a graphic model representation. By this way, we can measure the distance between nodes in the graphic model 83 which are corresponding to the words or concepts on ontology. Number of edges of the shortest path between two nodes is considered as the distance between them. As a result the shorter the distance is, the higher the semantic similarity is.

One of the simplest methods to adjust the distance [13] is to extend the minimum distance between the edge $\mathrm{cl}$ and $\mathrm{c} 2$ by the maximum depth $\mathrm{D}$ of a hierarchical classification system. For example, use only hyponymy- a kind of link between concepts.

$$
\operatorname{labSim}\left(c_{1}, c_{2}\right)=\max \left[-\log \left(\frac{\min _{c_{1}, c_{2}}\left[\operatorname{len}\left(c_{1}, c_{2}\right)\right]}{2} * D\right)\right]
$$

A method for calculating the similarity between a pair of concepts cl and c2 are presented in [14]. We determine the similarities based on the depth of the most super general concept $\mathrm{c} 3$ of $\mathrm{cl}$ and $\mathrm{c} 2$.

$$
\operatorname{labSim}\left(c_{1}, c_{2}\right)=\frac{2 * N 3}{N 1+N 2+2 * N 3}
$$

$\mathrm{N} 1$ is the length (number of nodes) of the path from cl to $\mathrm{c} 3$. N2 is the length of the path from $\mathrm{c} 2$ to $\mathrm{c} 3$, and N3 is the length of the path from c3 to the root directory of the hierarchy.

The similarity measurement between concepts / specific fuzzy attributes

Given a set of three parameters $\left\{\mathrm{A}, \mathrm{V}, f_{A}\right\}$ and $\left\{\mathrm{A}^{\prime}\right.$, $\left.\mathrm{V}^{\prime}, f_{A^{\prime}}\right\}$ belongs to the specific fuzzy concept/ attribute a and a' respectively. $\mathrm{V}$ and $\mathrm{V}$ ' is the specific sets. $A$ and $A^{\prime}$ are the corresponding fuzzy sets of sets $\mathrm{V}$ and $\mathrm{V}^{\prime}$. Get $U=V \cup V^{\prime}$, the operations on the fuzzy subsets of $A$ and $A^{\prime}$ are defined as follow:

$$
\begin{aligned}
& f_{A \cap A^{\prime}}=\min \left[f_{A}(x), f_{A^{\prime}}(x)\right], \forall x \in U \\
& f_{A \cup A^{\prime}}=\max \left[f_{A}(x), f_{A^{\prime}}(x)\right], \forall x \in U \\
& f_{A \backslash A^{\prime}}=\max \left[\min \left(f_{A}(x), 1-f_{A^{\prime}}(x)\right), \min (1-\right. \\
& \left.\left.f_{A}(x), f_{A^{\prime}}(x)\right)\right], \forall x \in U
\end{aligned}
$$

The attribute similarity in fuzzy ontology

$\operatorname{Sim}\left(a, a^{\prime}\right)=1-\left|A \backslash A^{\prime}\right|$

$\operatorname{Sim}\left(a, a^{\prime}\right)=1-\sup _{x \in U} f_{A \backslash A^{\prime}}(x)$

$\operatorname{Sim}\left(a, a^{\prime}\right)=\sup _{x \in U} f_{A \cap A^{\prime}}(x)$

The similarities between the concepts of fuzzy ontology Consider two fuzzy formal concepts $\left(c_{i}\right.$, $\left.A^{c_{i}}, V^{c_{i}}, f^{c_{i}}\right)$ and $\left(c_{j}, A^{c_{j}}, V^{c_{j}}, f^{c_{j}}\right)$ from two different 
fuzzy concepts. For $\mathrm{n}, \mathrm{m}$ is the number of the members of $c_{i}, c_{j}, n=\left|c_{i}\right|, m=\left|c_{j}\right|$, suppose that $\mathrm{n} \leq \mathrm{m}$. Set $\boldsymbol{T}\left(A^{c_{i}}, A^{c_{j}}\right)$ of the candidates from the pairs defined by all possible sets of $\mathrm{n}$ attribute pairs is defined as below:

$\left(A^{c_{i}}, A^{c_{j}}\right)=\{\{<\mathrm{a} 1, \mathrm{~b} 1>\ldots<\mathrm{an}, \mathrm{bn}\rangle\} \mid \mathrm{ah} \in A^{c_{i}}, \mathrm{bh} \in A^{c_{j}}$, $\forall \mathrm{h}=1 \ldots \mathrm{n}$, and $\left.\mathrm{ah} \neq a_{k}, b_{h} \neq b_{l}, \mathrm{l} \neq \mathrm{h}\right\}$

Consider an ontology domain, a concept called the similar concept from two fuzzy concepts $\left(c_{i}\right.$, $\left.A^{c_{i}}, V^{c_{i}}, f^{c_{i}}\right)$ và $\left(c_{j}, A^{c_{j}}, V^{c_{j}}, f^{c_{j}}\right)$ is defined as below:

\section{Similarity based on schema:}

$\operatorname{strSim}\left(\left(c_{i}\right),\left(c_{j}\right)\right)=$

$\frac{1}{\max \left(\left|A^{c_{i}}\right|,\left|A^{c_{j}}\right|\right)} \max _{P \in \mathcal{T}\left(A^{c_{i},} A^{c_{j}}\right)}\left[\sum_{\langle a, b\rangle \in P} f^{c_{i}} * f^{c_{j}} *\right.$

$\operatorname{labSim}(a, b)]$

Similarity based on instance:

$\operatorname{objSim}\left(\left(c_{i}\right),\left(c_{j}\right)\right)=\frac{\left|c_{i} \cap c_{j}\right|}{\max \left(\left|c_{i}\right|,\left|c_{j}\right|\right)}$

\section{Similarity based on instance and schema:}

$\operatorname{sSim}\left(\left(c_{i}\right),\left(c_{j}\right)\right)=\operatorname{objSim}\left(\left(c_{i}\right),\left(c_{j}\right)\right) * w+$

$\operatorname{strSim}\left(\left(c_{i}\right),\left(c_{j}\right)\right) *(1-w)$

Where:

- $f_{a}, f_{b}$ is the membership level of attributes a,b.

- $\mathrm{w}$ is the weight that $0 \leq \mathrm{w} \leq 1$, and the value is set by a user.

- $\operatorname{sim}_{L C}(\mathrm{a}, \mathrm{b})$ is the similarity value between a and $\mathrm{b}$.

\section{Constraint satisfaction problem for ontology matching}

Constraint programming $(\mathrm{CP})$ is an important technique in solving combinatorial optimization problems. Ontology matching is a process aimed at seeking semantic similarity between the components from two provided ontologies. Thus, constraint programming is a suited method to handle ontology matching. Moreover, it is a new approach to solve the constraint and optimization successfully. In this symposium, we only focus on resolving constraint satisfaction problems (CSPs) including: definitions for CSPs, constraints and solutions for CSPs.

\subsection{Definition 6: Ontology matching using CSP}

Given two ontologies: $O_{1}=\left(C_{1}, R_{1}, I_{1}, Z_{1}\right)$ and $O_{2}=$ $\left(C_{2}, R_{2}, I_{2}, Z_{2}\right)$, the matching problem must satisfy the constraint $\mathrm{P}$ consisting three parameters $P=$ $(C, D, B)$, where $\mathrm{C}=C_{1}=\left\{c_{1}^{1}, c_{2}^{1}, c_{3}^{1}, \ldots, c_{n}^{1}\right\}$ is a set of variables. $\mathrm{D}=\left\{D_{1}, D_{2}, \ldots, D_{n}\right\}$ is a set of corresponding value domain of each variable in $\mathrm{C}$. Each value domain $D_{i}=C_{2}=\left\{c_{1}^{2}, c_{2}^{2}, c_{3}^{2}, \ldots, c_{m}^{2}\right\}$ is the set of possible initial values for the corresponding variables $c_{i}^{1} \epsilon C$, and $\mathrm{B}=\left\{B_{1}, B_{2}, B_{3}, \ldots, B_{k}\right\}$ is finite set of constraints on the variables of $C$.

A constraint is previously defined as a pair consisting a set of variables and relationships. This definition gives us the basic background for modeling different types of fuzzy schema. In this paper, we need to define the similarity concept pairs between two fuzzy ontologies, called fuzzy ontology integration.

\subsection{Definition 7: Fuzzy constraint}

A fuzzy constraint $B_{\mu}$ on a set of variables $C=$ $\left(c_{1}, c_{2}, c_{3}, \ldots, c_{h}\right)$ is a pair $B_{\mu}=\left(\mathrm{C}, M_{\mu}\right)$ with a fuzzy relation $M_{\mu}$ determined by $\mu_{M}: \prod_{x_{i} \in \operatorname{var}(B)} D_{i} \rightarrow[0,1]$. $\mu_{M}$ is a membership function showing the degree which pair $v$ satisfies $B_{\mu} \cdot \mu_{M}(v)=1$ means that $v$ completely satisfies $B_{\mu} . \mu_{M}(v)=0$ says that $v$ violates $C_{\mu}$. $0<\mu_{M}(v)<1$ depicts that $v$ satisfies partial $B_{\mu}$.

\subsection{Definition 8: Fuzzy constraint optimization}

The problem of fuzzy constraint optimization $Q_{\mu}$ is a set of four parameters $Q_{\mu}=\left(C, D, B_{\mu}, g\right)$ with $\mathrm{C}$ is a list of concepts on ontology $O_{1}$, D is a list of concepts on ontology $\mathrm{O}_{2}$ which can be matched to the concepts in $\mathrm{C}, B_{\mu}$ is a list of fuzzy constraints where each one mentions to one or more concepts in $C$ and $D . \mathrm{g}$ is the optimized objective function.

\section{Constraint construction \\ 5.1Relation constraint}

If there exists a relation $r$ between two concepts $c_{i}, c_{j} \in C \cup D$, there will exist their corresponding mapping as below:

With

$$
\begin{aligned}
& B_{M_{\mu}\left(c_{i}, c_{j}\right)}^{\text {relation }} \\
& =\left\{R_{1} \cup R_{2}\right. \\
& \cup\left\{\left(c_{i}, c_{j}\right) \in(C \cup D) \times(C \cup D) \mid \exists r\left(c_{h}, c_{k}\right) \in R_{1} \cup R_{2}\right\} \\
& \wedge\left(\left(c_{k}, c_{j}\right),\left(c_{h}, c_{i}\right) \in C_{M_{\mu}(v)}^{\text {parent }}\right) \vee\left(c_{i} \in B_{M_{\mu}\left(c_{h}\right)}^{L a b}\right. \\
& \left.\cup B_{M_{\mu}\left(c_{h}\right)}^{\text {obj }} \cup B_{M_{\mu}\left(c_{h}\right)}^{s t r} \cup B_{M_{\mu}\left(c_{h}\right)}^{r e l}\right) \wedge\left(c_{j} \in B_{M_{\mu}\left(c_{k}\right)}^{L a b}\right. \\
& \left.\left.\cup B_{M_{\mu}\left(c_{k}\right)}^{o b j} \cup B_{M_{\mu}\left(c_{k}\right)}^{s t r} \cup B_{M_{\mu}\left(c_{k}\right)}^{r e l}\right)\right\}
\end{aligned}
$$

Every pair of two concepts $c_{i}, c_{j} \in C \cup D$, there exists a set of binary constraints satisfied: 
- Parent constraint: $B_{M_{\mu}\left(c_{i}, c_{j}\right)}^{\text {parent }}$ represent the relation

Parent - Child from $c_{i} \rightarrow c_{j}$, as following:

$$
\begin{aligned}
B_{M_{\mu}\left(c_{i}, c_{j}\right)}^{\text {parent }}=\left\{\left(c_{i}, c_{j}\right)\right. & \\
& \in(C \cup D) \times(C \\
& \left.\cup D) \mid \exists\left(c_{i}, c_{j}\right) c_{j} \text { isa } c_{i}\right\}
\end{aligned}
$$

- Child constraint: $B_{M_{\mu}\left(c_{i}, c_{j}\right)}^{\text {child }}$ represents the relation

Child - Parent from $c_{j} \rightarrow c_{i}$, as following:

$$
\begin{aligned}
B_{M_{\mu}\left(c_{i}, c_{j}\right)}^{\text {child }}=\left\{\left(c_{i},\right.\right. & \left.c_{j}\right) \\
& \in(C \cup D) \times(C \\
& \left.\cup D) \mid \exists\left(c_{i}, c_{j}\right) c_{i} \text { isa } c_{j}\right\}
\end{aligned}
$$

- Sibling constraint: $B_{M_{\mu}\left(c_{i}, c_{j}\right)}^{s i b l}$ represents the sibling relation as following:

$$
\begin{array}{r}
B_{M_{\mu}\left(c_{i}, c_{j}\right)}^{\text {sibl }}=\left\{\left(c_{i}, c_{j}\right) \in(C \cup D) \times(C \cup D) \mid \exists\left(c_{i}, c_{h}\right)\right. \\
\left.\in B_{M_{\mu}\left(c_{i}, c_{j}\right)}^{\text {child }} \wedge\left(c_{j}, c_{h}\right) \in B_{\mu_{M}\left(c_{i}, c_{j}\right)}^{\text {child }}\right\}
\end{array}
$$

5.2Similarity based on vocabulary

For every concept $c_{i}^{1} \in C$, there exists a constraint $B_{M_{\mu}\left(x_{i}\right)}^{L a b}$ ensuring the semantic similarity between $c_{i}^{1} \in C$ và $c_{j}^{2} \in D_{i}$ through the linguistic meaning similarity of corresponding labels:

$B_{\mu_{M}\left(c_{i}^{1}\right)}^{L a b}=$

$\left\{c_{j}^{2} \in D_{i} \mid \operatorname{labSim}\left(\operatorname{lab}\left(c_{i}^{1}\right), \operatorname{lab}\left(c_{j}^{2}\right)\right) \geq \theta\right\}$ with $c_{i}^{1} \in$

C

Where

labSim is a semantic similarity between the labels of the concepts and $\theta$ is a pre-defined threshold.

\subsection{Similarity based on instance}

For every concept $c_{i}^{1} \in C$, there exists a constraint $B_{M_{\mu}\left(x_{i}\right)}^{O b j}$ ensuring the semantic similarity between $c_{i}^{1} \in C$ và $c_{j}^{2} \in D_{i}$ which is demtermined by common instances.

$B_{\mu_{M}\left(c_{i}^{1}\right)}^{o b j}=\left\{c_{j}^{2} \in D_{i} \mid \operatorname{objSim}\left(\left(c_{i}^{1}\right),\left(c_{j}^{2}\right)\right) \geq \theta\right\}$ with

$c_{i}^{1} \in C$

\subsection{Similarity based on schema}

For every concept $c_{i}^{1} \in C$, there exists a constraint $B_{M_{\mu}\left(x_{i}\right)}^{O b j}$ ensuring the semantic similarity between $c_{i}^{1} \in C$ và $c_{j}^{2} \in D_{i}$ which relies on the similarity of correlative attributes.

$$
\begin{aligned}
& B_{\mu_{M}\left(c_{i}^{1}\right)}^{s t r}= \\
& \left\{c_{j}^{2} \in D_{i} \mid \operatorname{str} \operatorname{Sim}\left(\left(c_{i}^{1}\right),\left(c_{j}^{2}\right)\right) \geq \theta\right\} \text { with } c_{i}^{1} \in C
\end{aligned}
$$

\subsection{Similarity based on classification}

For every concept $c_{i}^{1} \in C$, there exists a constraint $B_{M_{\mu}\left(x_{i}\right)}^{O b j}$ ensuring the semantic similarity between $c_{i}^{1} \in C$ và $c_{j}^{2} \in D_{i}$ which is determined by their sibling structure similarity.

$B_{\mu_{M}\left(c_{i}^{1}\right)}^{r e l}=$
$\left\{c_{j}^{2} \in D_{i} \mid \operatorname{relSim}\left(\left(c_{i}^{1}\right),\left(c_{j}^{2}\right)\right) \geq \theta\right\}$ with $c_{i}^{1} \in C$

The syntax and semantics constraints depend on a membership function $M_{\mu}(v) \in[0,1]$ which refers to an acceptable satisfaction level. If $M_{\mu}(v)=0$, this represents $\mathrm{v}$ violates all constraints and $M_{\mu}(v)=1$ shows $v$ completely satisfies the pre-defined constraints. These constraints limit the search space of integration, which could lead to a highly efficient search process. On the other hand, if the problem is too complex, the constraints will produce complicated computations to support the solutions. Finally, $v$ can be a relationship between concepts or a specific concept.

\subsection{Objective function}

The objective function is aimed at minimizing the matching errors via constraint satisfaction level and finds the number of matching through total elements assigned to variables. With that meaning, we have

$$
f_{\text {optimazation }}=\sum_{i=1 . . k} M_{\mu}\left(v_{i}\right) / \sum_{i=1 . . n}\left|c_{i}^{1}\right|
$$

Where

$M_{\mu}(v)$ is a membership function.

$M_{\mu}(v) \in[0,1]$ refers to the acceptable satisfaction level by an assignment operation $v$ to constraints.

$\left|c_{i}^{1}\right|$ is the total number of constraints.

\section{Experiment}

6.1Dataset

According to the aforementioned fuzzy ontology definition, we propose a fuzzy weather Ontology construction process and apply fuzzy description logic into OWL. First, we use the software protégé 5.0 to produce fuzzy weather ontology structure. Then we add the description to fuzzy ontology based on fuzzy description logic by using the annotation property. Next, we conduct the description syntax interpretations from OWL to the languages which are supported by some other fuzzy DL reasoners in order to determine the suitability of our fuzzy OWL. Here we build five fuzzy ontologies by reusing predefined weather ontologies [1], the main concepts are as shown in Table 1. 
Xuan Hung Quach et al.

Table 1 The main concepts in weather ontologies

\begin{tabular}{|c|c|c|c|}
\hline Concept & Sub-concept & Attributes & Range \\
\hline \multirow[t]{4}{*}{ Cloud cover } & & hascloudcover & m \\
\hline & Partly cloudy & & $1,2,3,4$ \\
\hline & Mostly cloudy & & $5,6,7$ \\
\hline & Overcast & & 8,9 \\
\hline \multirow[t]{4}{*}{ Humidity } & & hasHumidity & - \\
\hline & Dry & & {$[0.3 ; 0.4)$} \\
\hline & Average humidity & & {$[0.4 ; 0.7]$} \\
\hline & Moist & & $(0.7 ; 0.8]$ \\
\hline \multirow[t]{4}{*}{ Rain } & & hasPrecipitationRate & $\mathbf{M m} / \mathbf{h}$ \\
\hline & Light rain & & {$[2.5,5]$} \\
\hline & Moderate rain & & $(5,5,7.5]$ \\
\hline & Heavy rain & & $>7.5$ \\
\hline \multirow[t]{9}{*}{ Wind } & & hasWindSpeed & $\mathbf{m} / \mathbf{s}$ \\
\hline & Light wind & & $(5,20]$ \\
\hline & Medium wind & & $(20,40]$ \\
\hline & Heavy wind & & $>40$ \\
\hline & & hasWindDirection & $\mathbf{o}$ \\
\hline & North wind & & {$[0 ; 45)[315 ; 360)$} \\
\hline & East wind & & {$[45 ; 135)$} \\
\hline & South wind & & {$[135 ; 225)$} \\
\hline & West wind & & {$[225 ; 315)$} \\
\hline
\end{tabular}

The mismatching concepts between ontologies by their labels and relations are created for demonstration. These mismatching concepts have the same name but are different concepts or include some conflicting hierarchical relations.

\subsection{Result}

We use the algorithm proposed by [1] to do the matching process between weather ontologies. In this beginning experiment, we do not consider constraints between concepts (denoted as BCS) using the previously proposed algorithm [1], but the output result is used for refinement by applying the constraint optimization problem (denoted as FCS). The comparison using precision measurement is shown in Figure 1.

From the result we can conclude that using constraint optimization is a significantly effective approach for ontology matching refinement.

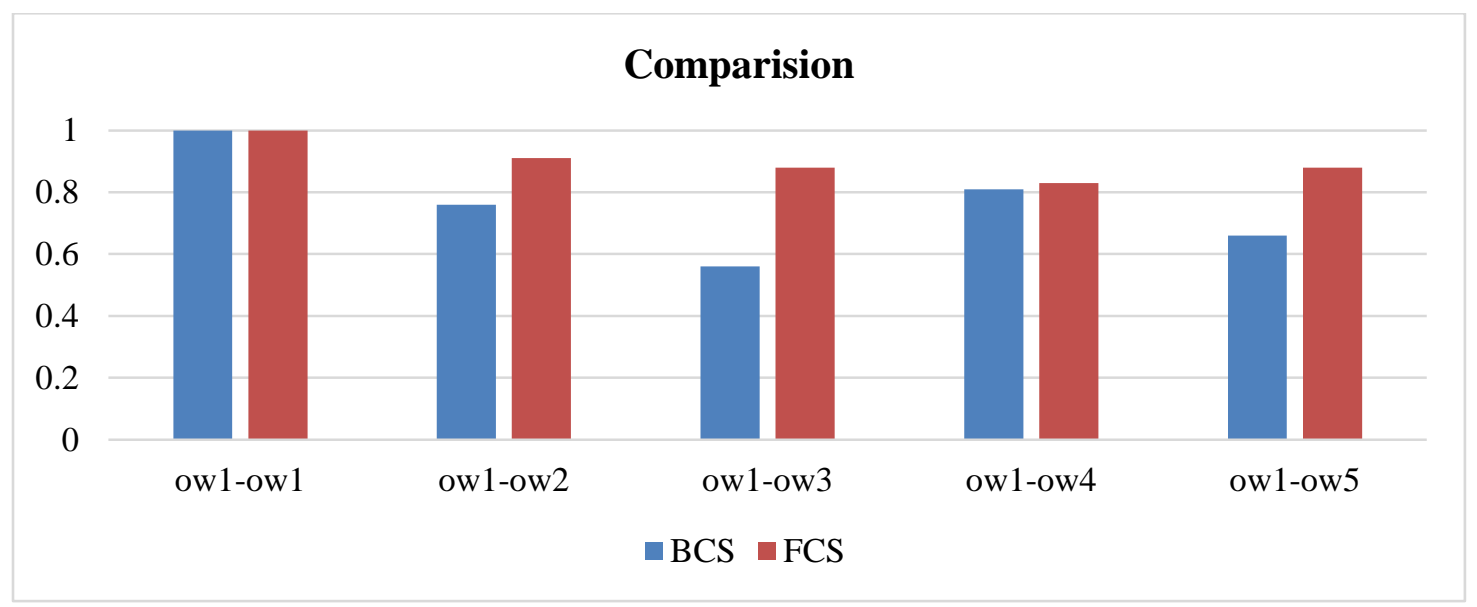

Figure 1 The comparison between BCS and FCS

\section{Conclusion}

The main contribution of this work is using CSP for fuzzy ontology matching integration. In particular, a 86 fuzzy ontology model is much enhanced from our previous works [8] by adding fuzzy membership to a concept. This is useful for reasoning in a fuzzy 
inference system such as weather forecasting. We proposed a fuzzy ontology integration using CPS to refine the mismatching. However, the optimal function for minimizing mismatching should be improved to consider all aspects during the matching execution. This direction also navigates us for our future work to ameliorate the performance of this integration problem.

\section{Acknowledgment}

None.

\section{Conflicts of interest}

The authors have no conflicts of interest to declare.

\section{References}

[1] Truong HB, Duong TH, Nguyen NT. A hybrid method for fuzzy ontology integration. Cybernetics and Systems. 2013; 44(2-3):133-54.

[2] Todorov K, Geibel P, Hudelot C. A framework for a fuzzy matching between multiple domain ontologies. In international conference on knowledge-based and intelligent information and engineering systems 2011 (pp. 538-47). Springer Berlin Heidelberg.

[3] Chen RC, Bau CT, Yeh CJ. Merging domain ontologies based on the WordNet system and fuzzy formal concept analysis techniques. Applied Soft Computing. 2011; 11(2):1908-23.

[4] Duong TH, Truong HB, Nguyen NT. Local neighbor enrichment for ontology integration. In Asian conference on intelligent information and database systems 2012 (pp. 156-66). Springer Berlin Heidelberg.

[5] Truong HB, Nguyen NT. A framework of an effective fuzzy ontology alignment technique. In IEEE international conference on systems, man, and cybernetics 2011 (pp. 931-5). IEEE.

[6] Truong HB, Nguyen NT. A multi-attribute and multivalued model for fuzzy ontology integrationon instance level. In Asian conference on intelligent information and database systems 2012 (pp. 187-97). Springer Berlin Heidelberg.

[7] Truong HB, Nguyen NT, Nguyen PK. Fuzzy ontology building and integration for fuzzy inference systems in weather forecast domain. In Asian conference on intelligent information and database systems 2011 (pp. 517-27). Springer Berlin Heidelberg.

[8] Truong HB, Quach XH. An overview of fuzzy ontology integration methods based on consensus theory. In advanced computational methods for knowledge engineering 2014 (pp. 217-27). Springer International Publishing.
[9] Song L, Ma J, Liu H, Lian L, Zhang D. Fuzzy semantic similarity between ontological concepts. In advances and innovations in systems, computing sciences and software engineering 2007 (pp. 275-80). Springer Netherlands.

[10] Cross VV, Voss C. Fuzzy ontologies for multilingual document exploitation. In international conference of the North American fuzzy information processing society 1999. (pp. 392-7). IEEE

[11] Cross V. Fuzzy semantic distance measures between ontological concepts. In IEEE annual meeting of the fuzzy information 2004 (pp. 635-40). IEEE.

[12] Rada R, Mili H, Bicknell E, Blettner M. Development and application of a metric on semantic nets. IEEE Transactions on Systems, Man, and Cybernetics. 1989; 19(1):17-30.

[13] Leacock C, Chodorow M. Combining local context and WordNet similarity for word sense identification. WordNet: An Electronic Lexical Database. 1998; 49(2):265-83.

[14] Calegari S, Loregian M. Using dynamic fuzzy ontologies to understand creative environments. In international conference on flexible query answering systems 2006 (pp. 404-15). Springer Berlin Heidelberg.

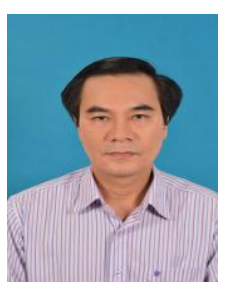

Xuan Hung Quach received the Master degree in Computer Science from the Department of Information Technology of the Science University, Hue University, Vietnam, in 2007. His is currently Doctoral study at the Department of Information Technology of the Science University, Hue University, Vietnam. His research interests are in ontology integration and semantic web.

Email: tiasang70@yahoo.com

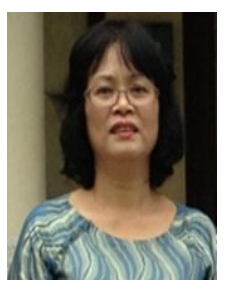

Lan Giao Hoang Thi received her Ph.D. degree in Computer Science from the Institute of Information and Technology, Viet Nam Academy of Science and Technology, Vietnam in 2008. Her is currently Lecturer at the Department of Information Technology of the Science University, Hue University, Vietnam. Her research interests include artificial intelligence, expert system, data mining and raw set theory. 